\title{
Enseñanza y divulgación de la ciencia en la integración universidad-escuela: una experiencia en Brasil
}

\author{
Cássio C. Laranjeiras ${ }^{1, a}$, Sebastião I. C. Portela ${ }^{2, b}$, Luiz A. Ribeiro ${ }^{1, c}$ \\ ${ }^{1}$ Instituto de Física. Universidade de Brasilia. Brasília, DF. Brasil. \\ ${ }^{2}$ Centro de Ensino Médio Integrado à Educação Profissional (CEMI). Gama, Brasilia, DF. Brasil. \\ acassio@unb.br,bprofsebastiao@yahoo.com.br,cribeirojr.fis@gmail.com
}

[Recibido: 12 Julio 2017. Revisado: 24 Septiembre 2017. Aceptado: 23 Diciembre 2017]

\begin{abstract}
Resumen: A partir de la idea de que la Ciencia y la Tecnología desempeñan un papel central en nuestra lectura y comprensión del mundo, identificamos la centralidad y urgencia de la educación científica en la formación del ciudadano contemporáneo, defendiendo la necesidad de la integración universidad-escuela en acciones orientadas hacia la comprensión pública de la ciencia por los diferentes sectores de la sociedad. Se presentan aquí algunas experiencias desarrolladas en Brasil por el Instituto de Física de la Universidad de Brasilia, a través de su Núcleo de Investigación en Enseñanza de la Física (NPEF), en asociación con algunas escuelas públicas de la capital federal y el Museo de la Ciencia y Tecnología de Brasilia (en implantación).
\end{abstract}

Palabras clave: Enseñanza de la ciencia; Divulgación de la ciencia; Integración universidad-escuela; Museo de ciencia.

Teaching and science divulgation in university-school integration: an experience in Brazil

Abstract: Based on the idea that science and technology play a central role in our reading and understanding of the world, we identify the centrality and urgency of scientific education in the formation of the contemporary citizen, defending the need for university-school integration in actions oriented towards the public understanding of science by the different sectors of society. Some of the experiences developed in Brazil by the Institute of Physics of the University of Brasilia, through its Physics Education Research Group, in association with some public schools of the federal capital and the Museum of Science and Technology of Brasilia (in implementation), are presented here.

Keywords: Science education; Science divulgation; University-school integration; Science museum.

Para citar este artículo: C. C. Laranjeiras, S. I. C. Portela, L. A. Ribeiro (2018) Enseñanza y divulgación de la ciencia en la integración universidad-escuela: una experiencia en Brasil. Revista Eureka sobre Enseñanza y Divulgación de las Ciencias 15 (2), 2201 doi:10.25267/Rev_Eureka_ensen_divulg_cienc.2018.v15.i2.2201

\section{Introducción}

Las realizaciones y conquistas del mundo contemporáneo, traducidas entre otros aspectos en la modernización de los medios de producción y en la utilización cotidiana de bienes y servicios basados en estándares científicos y tecnológicos cada vez más perfeccionados, han aumentado el nivel de exigencia de conocimientos de los ciudadanos con relación a la ciencia y la tecnología. Recordando aquí al astrónomo americano y gran divulgador de la ciencia Carl Sagan 1997): «Hemos organizado una civilización global en la que los elementos más cruciales (...) dependen profundamente de la Ciencia y de la Tecnología».

Esta realidad plantea inmensos retos a la educación científica (formal y no formal), que se ve ante la necesidad de proporcionar, sobre todo, a los más desfavorecidos económica y socialmente, un conjunto de conocimientos básicos necesarios para su inclusión social. En esta dirección, los programas de educación, divulgación y popularización de la ciencia y de la tecnología asumen un relevante papel, promoviendo la cultura científica y tecnológica a la condición de partes integrantes esenciales a la formación del ciudadano contemporáneo. Así, el concepto de alfabetización cientifica (McConney, Oliver, Woods-McConney, Schibeci y Maor 
2014) se ha convertido en un objetivo internacional clave en el enfrentamiento de los actuales desafíos de la humanidad (OCDE 2016).

Conscientes de esta realidad, el Instituto de Física de la Universidad de Brasilia, a través de su Núcleo de Investigación en Enseñanza de la Física (NPEF), en asociación con algunas escuelas públicas de la capital federal y el Museo de Ciencia y Tecnología de Brasilia, busca desarrollar acciones integradas en el campo de la educación, divulgación y popularización de la ciencia y la tecnología.

Además de una breve contextualización histórica de la enseñanza y la divulgación de la ciencia en Brasil, se presentan en las siguientes secciones un relato de la experiencia desarrollada.

\section{Contextualización y desafíos de la enseñanza y la divulgación de la ciencia en el Brasil}

Aunque la enseñanza y la divulgación de la ciencia en el Brasil tengan al menos dos siglos de historia, iniciada con la transferencia de la Corte portuguesa a Brasil en 1808 (Massarani y Moreira 2002), esta actividad continúa desafiándonos de manera intensa y compleja.

Según Massarani y Moreira (2002, pp. 44-45) «la primera manifestación más consistente de actividades divulgativas en el Brasil vendría a ocurrir a principios del siglo XIX. Se derivó de una razón política imperativa: con la llegada de la Corte portuguesa al país, se abrieron los puertos y la prohibición de imprimir fue suspendida. Poco después, surgieron las primeras instituciones de enseñanza superior o con algún interés ligado a la ciencia y las técnicas, como la Academia Real Militar (1810) y el Museo Nacional (1818)».

En aquella época, importantes transformaciones políticas, culturales y económicas, que Brasil pasó a protagonizar, promovieron, además de la creación de las primeras instituciones ligadas a la ciencia, la publicación de libros y el acceso a las novedades del viejo continente. Datan de ese período las primeras publicaciones de textos y manuales -traducciones francesas en su casi totalidad- dedicados a la educación científica, destacando las áreas de medicina e ingeniería. Posteriormente -ya en la segunda mitad del siglo XIX, como fruto de una intensificación de la divulgación de la ciencia en el mundo y del interés por la ciencia demostrada por don Pedro II, emperador de Brasil en la época- algunas acciones favorecieron la divulgación de la ciencia en Brasil, sobre todo con la publicación de diferentes revistas dedicadas al público general (Massarani y Moreira 2015).

Las primeras décadas del siglo xx fueron marcadas por un movimiento en favor de la ciencia pura, con la creación de importantes instituciones científicas, destacando el papel desempeñado por la Academia Brasileña de Ciencias (1921), que prontamente inició acciones en el campo de la divulgación y popularización de la ciencia (Massarani y Moreira 2015, p. 5).

Aunque muchas cosas han ocurrido desde aquella época, acciones significativas buscando consolidar políticas públicas en el área, en consonancia con procesos sistemáticos de educación científica, surgieron solamente en las últimas dos décadas: creación de centros y museos de ciencias, edición de revistas científicas dirigidas al público que incluye el público infantil, eventos locales, regionales y nacionales orientados a la divulgación de la ciencia, creación de planetarios, olimpiadas científicas, proyectos itinerantes de ciencia, etc.

Si, por un lado, no hay duda de que estas son acciones fundamentales, por otro, se hace necesario planificarlas cuidadosamente para que su ampliación, multiplicación y potenciación puedan de hecho atender las reales demandas de la educación científica de un país de dimensiones continentales, como es el caso de Brasil, que reúne 26 estados federados aún bastante desiguales económica y socialmente. La preocupación se justifica también desde el 
punto de vista pedagógico. Y aquí es importante tener mucho cuidado para evitar acciones donde el conocimiento científico sea reducido a un instrumento de «espectacularización», de mero entretenimiento, de simple mecanismo de consumo, que más adormecen de lo que promueven la conciencia, reduciendo así la significancia de tan importante proceso educativo. ${ }^{1}$ La observación nos parece importante, pues, aunque, defendiendo y asumiendo el papel fundamental de la dimensión lúdica y placentera en los procesos de enseñanza, aprendizaje y divulgación de la ciencia -sobre todo en los primeros contactos con la ciencia-, reconocemos la imperiosa necesidad de no confundirlas con prácticas cuyo objetivo es el entretenimiento.

En esa dirección, la asociación entre las universidades -cuyos objetivos involucran actividades de enseñanza, investigación y extensión- y las escuelas de la red pública de enseñanza de educación básica puede ser de gran valor.

Con el surgimiento en 2004 de un programa gubernamental brasileño de incentivo y fomento a proyectos itinerantes de divulgación científica fue posible iniciar la experiencia que aquí relatamos. En esa ocasión, el Ministerio de Ciencia, Tecnología e Innovación (MCTI) del gobierno brasileño y la Academia Brasileña de Ciencias (ABC) lanzaron una convocatoria pública para fomentar proyectos itinerantes de divulgación científica. Nueve proyectos fueron contemplados (incluyendo el nuestro), lo que fue fundamental para fortalecer el modelo en el país. La gran referencia para esta iniciativa fue el Proyecto de Museo Itinerante (Promusit) de la Pontificia Universidad Católica de Río Grande do Sul (PUC-RS), Brasil, creado en 2001 por el biólogo Jeter Bertoletti. El Promusit fue el primer museo itinerante de Brasil.

A continuación se presentan algunas experiencias desarrolladas por el Instituto de Física de la Universidad de Brasilia, desde el 2005 hasta el presente momento, a través de su Núcleo de Investigación en Enseñanza de la Física (NPEF), en asociación con algunas escuelas públicas de la capital federal y el Museo de la Ciencia y Tecnología de Brasilia. ${ }^{2}$ Estas experiencias nos han ayudado a construir una perspectiva de educación científica donde la integración Universidad-Escuela se ha mostrado prometedora.

\section{Educación científica en acción}

Uno de nuestros frentes de acción consiste en llevar actividades científico-culturales dialógicas e investigativas como parte de Ferias de Ciencias o Muestras Científico-Culturales realizadas en escuelas de la red pública de enseñanza de la educación básica y en lugares públicos. Para ello utilizamos dos unidades móviles (como se muestra en la figura 1), que nos permiten transportar un conjunto de objetos pedagógicos denominados Objetos Científicos Interactivos (OCI) y un Planetario Inflable.

Las actividades cuentan siempre con la participación de estudiantes de graduación o postgrado del curso de Física, estudiantes del curso de Ingeniería y de alumnos de la enseñanza media. Estos estudiantes actúan como mediadores del proceso educativo, que busca siempre la interacción con los proyectos desarrollados en el ámbito de las escuelas de educación básica asociadas en la acción.

La organización de las actividades, montadas en forma de exposiciones científicas interactivas, se hace siempre en conjunto con las escuelas asociadas, en encuentros que anteceden a la acción propiamente dicha. En estos encuentros se definen los OCI que serán utilizados en la acción, buscando siempre compatibilizarse con la temática abordada por el profesor en aquel

\footnotetext{
${ }^{1}$ Esta perspectiva de la conciencia en la relación con el conocimiento, que hace el intento de comprensión del mundo una actitud crítica y reflexiva -inspirada en el pensamiento educativo de Paulo Freire (2016) - es algo que parece perderse, o al menos sí diluir, al utilizar la estrategia de la «espectacularización», del mero entretenimiento. ${ }^{2}$ En estos 12 años del proyecto se realizaron cerca de 720 visitas, atendiendo en promedio a 50 escuelas y 250 profesores, en un total aproximado de 36.000 alumnos de educación primaria y secundaria.
} 
momento. El equipo del proyecto cuenta también con profesores de esas escuelas -algunos de ellos ex alumnos del curso de licenciatura impartidos por la Universidad-que siempre tienen participación activa en el proceso de mediación.
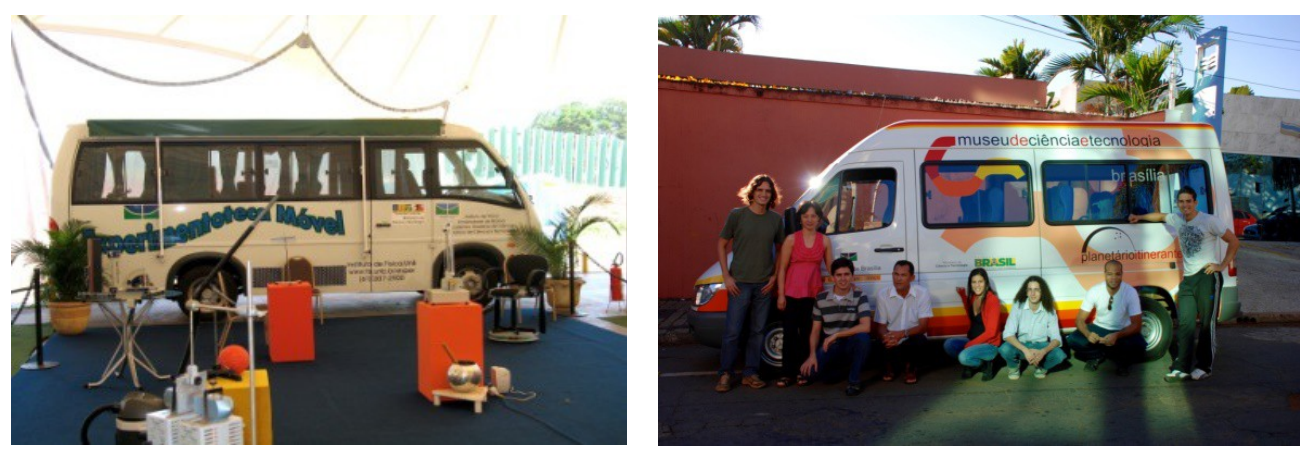

Figura 1. Experimentoteca móvil (izquierda) y Planetario Itinerante (derecha).

Las actividades se realizan también en lugares públicos para atender a un público en general. En este caso, como no hay compromiso con estructuras curriculares, la temática explotada se muestra variada, aunque la metodología de trabajo centrada en el estímulo a la curiosidad y al espíritu de investigación de los participantes se mantiene. En este caso, para evitar que las actividades sean simplemente de carácter demostrativo o ilustrativo, invitamos a los participantes a elegir uno de los OCI (el que más le haya despertado la atención e interés) para un diálogo más profundo.

A lo largo de una década de acciones continuas, hemos identificado un interés cada vez mayor, sobre todo, del público general por actividades relacionadas con la ciencia y la tecnología, lo que plantea fuertes desafíos en el campo de la comunicación pública de la ciencia e indica la necesidad urgente de profesionalización de las acciones de mediación en este campo. ${ }^{3}$ En este contexto, buscamos desarrollar materiales pedagógicos que pudieran potenciar el proceso comunicativo con el público general, preservando al mismo tiempo características esenciales de la investigación científica. Así nacieron los llamados Objetos Científicos Interactivos (OCI).

\section{Los objetos científicos interactivos (OCI) en la enseñanza y divulgación de la ciencia}

Los Objetos Científicos Interactivos (OCI) son objetos manipulables que incorporan intencionalidades didáctico-pedagógicas en su construcción y utilización. Se caracterizan por la integración de tres dimensiones fundamentales a la enseñanza formal de la ciencia y también esenciales para su proceso de comunicación pública: dialogicidad, ludicidad e interactividad. Por esas características los OCI son capaces de movilizar al público, despertando en ellos la curiosidad y el espíritu de investigación. En la figura 2, presentamos algunos ejemplos de OCI desarrollados por nosotros y utilizados en nuestras actividades.

En su estructura conceptual, un OCI se concibe como una articulación de tres elementos básicos: objeto, fenómeno y contexto. El objeto se constituye en el elemento sobre el cual y a partir del cual los fenómenos ocurren, corporificándose por medio de una base material, que ocupa espacio se presenta en forma de equipos, prototipos, instalaciones artísticas, minerales, fósiles, vegetales, animales y otros, o una asociación de éstos. El fenómeno, a su vez, más que simple base material, incorpora y reivindica una «lectura» -una aprehensión de la experiencia según nuestros esquemas mentales- que explicite diferentes conexiones de un determinado evento

\footnotetext{
${ }_{3}^{3}$ Aunque exista en el proyecto un trabajo de capacitación y formación de mediadores, basado sobre todo en el intercambio de experiencia entre ellos, reconocemos que todavía se muestra tímido y limitado para las exigencias de la propuesta de trabajo.
} 
en el tiempo. ${ }^{4}$ Sin embargo, la relación objeto-fenómeno no ocurre en el vacío, lo que hace que sea necesario explicitar un contexto, capaz de albergar y al mismo tiempo traducir la relación objeto-fenómeno, confiriéndole un sentido. A raíz de esta perspectiva de contextualización, se encuentra un entendimiento acerca de la naturaleza de la ciencia, en sus diferentes dimensiones constitutivas.
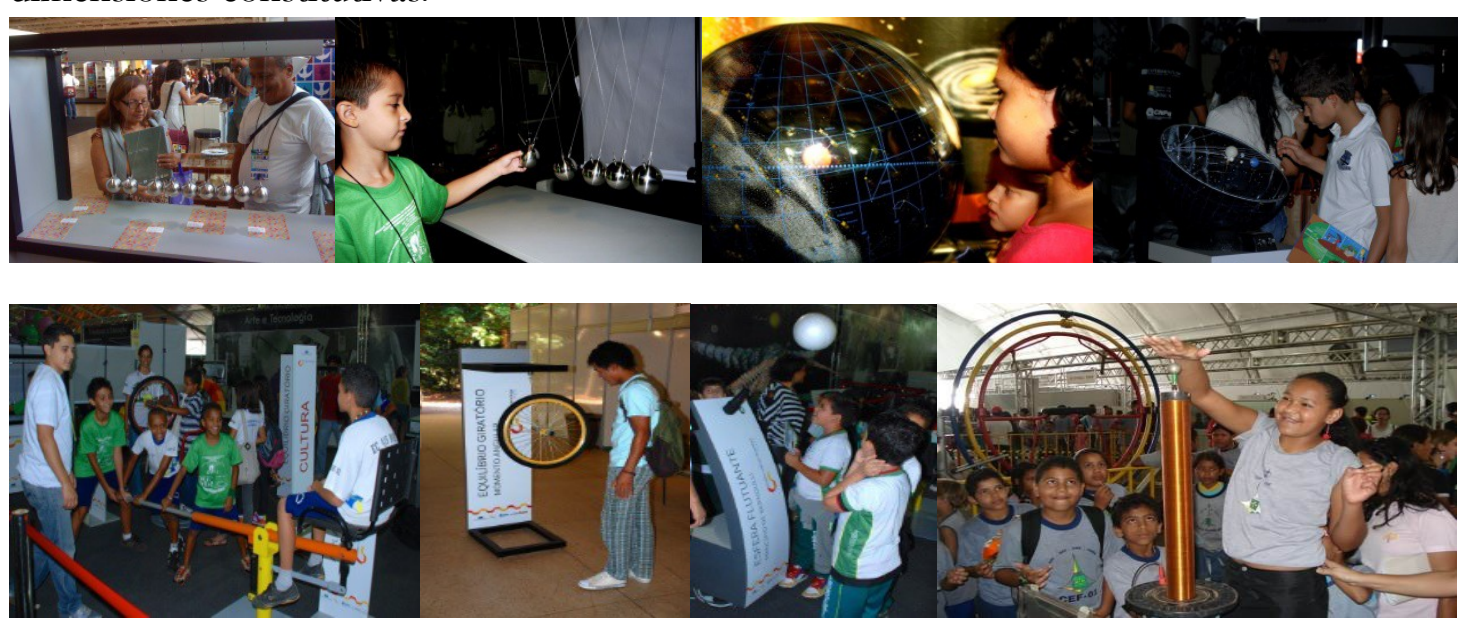

Figura 2. Algunos ejemplos de Objetos Científicos Interactivos (OCI), utilizados en nuestras actividades.

En la figura 3 tenemos un ejemplo de utilización de una réplica de un fósil como un OCI, articulando objeto, fenómeno y contexto en una acción educativa desarrollada en una exposición científica. Se trata de un Unaysaurus tolentinoi, un fósil de una especie de dinosaurio herbívoro que vivió hace aproximadamente entre 225 y 200 millones de años, al final del período Triásico, y que fue encontrado en el estado de Río Grande del Sur, Brasil.
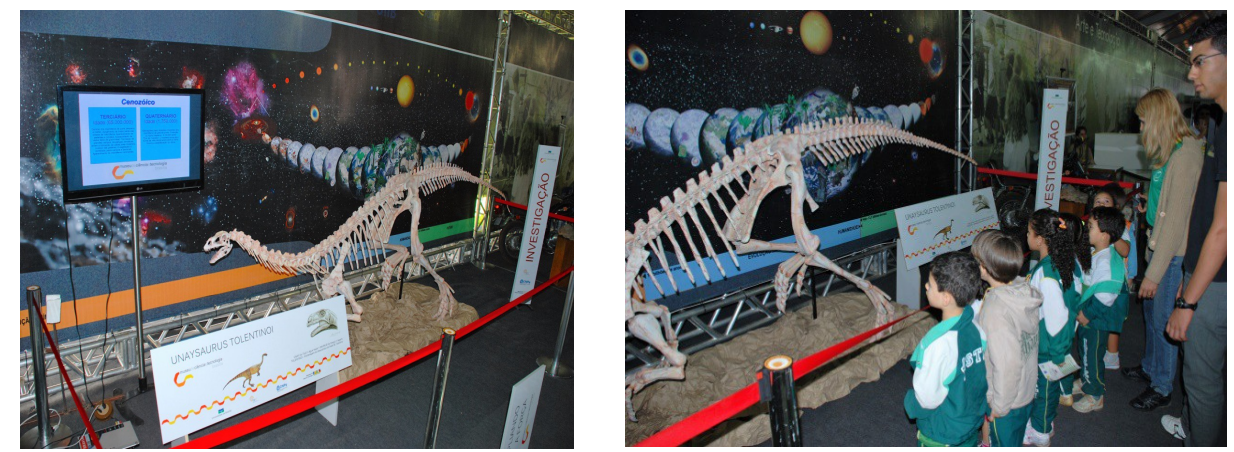

Figura 3. Réplica de un fósil de un dinosauro (Unaysaurus tolentinor) en una exposición científica.

En la figura 3 tenemos un ejemplo de utilización de una réplica de un fósil como un OCI, articulando objeto, fenómeno y contexto en una acción educativa desarrollada en una exposición científica. Se trata de un Unaysaurus tolentinoi, un fósil de una especie de dinosaurio herbívoro que vivió hace aproximadamente entre 225 y 200 millones de años, al final del período Triásico, y que fue encontrado en el estado de Río Grande del Sur, Brasil.

La réplica del fósil se constituye inicialmente como mero objeto; el diálogo en torno a él (y «con él») nos permite aprehenderlo (incluso como réplica) como fenómeno -en este caso específico, como registro de la evolución biológica en la Tierra, posibilitando más allá de la datación, la reconstitución de la historia geológica del planeta. Es esa «lectura» que aquí caracterizamos como fenómeno; el contexto nos permite explicitar la dinámica intrínseca de la producción de conocimiento en torno a aquel fósil (en el caso específico, una réplica) en especial.

\footnotetext{
${ }^{4}$ El uso de las categorías «objeto» y «fenómeno» en el diseño de los Objetos Científicos Interactivos (OCI) está inspirado en las ideas del físico y divulgador de la ciencia español Jorge Wagensberg (2005).
} 
El punto de partida es siempre una cuestión o un problema, a partir del cual la ciencia es, en cuanto conocimiento, movilizada con miras a la búsqueda de solución, traduciendo de esta manera el espíritu científico, en la perspectiva de Gastón Bachelard (1986), para el cual «todo conocimiento es una respuesta a una pregunta».

Tomamos también como fundamento la perspectiva pedagógica y epistemológica del educador brasileño Paulo Freire, de que «todo conocimiento es una cooperación» (Freire 1973). En este sentido, el diálogo encuentra su lugar (privilegiado) en el proceso de movilización intelectual de los interlocutores, lugar como elemento constitutivo de la promoción de la conciencia en la relación con el conocimiento.

\section{La asociación universidad-escuela en acción}

En las figuras 4 y 5, como ejemplos de la participación activa del público en situaciones de aprendizaje -mediadas por OCI- se ven estudiantes de enseñanza fundamental y también algunos adultos, dialogando en torno a situaciones desafiantes.
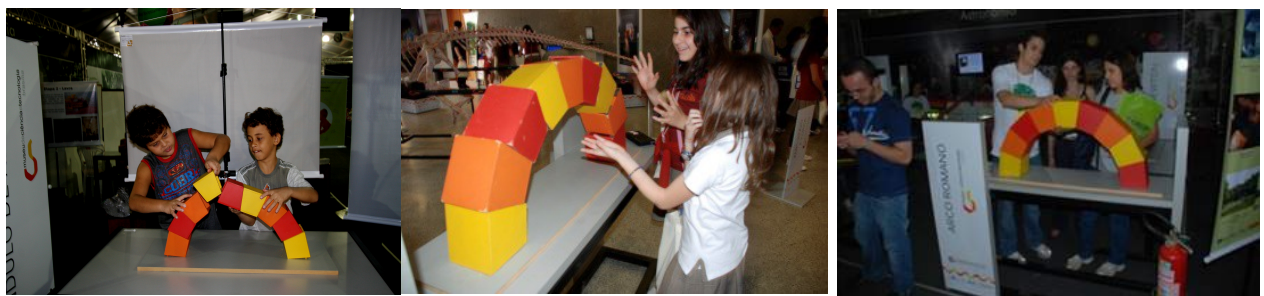

Figura 4. Alumnos de la educación básica (izquierda y centro) y algunos adultos (derecha) en situaciones de aprendizaje investigativo.

En la figura 4 (izquierda y centro) estudiantes de enseñanza primaria trabajan conjuntamente en el montaje de un modelo de arco romano, ${ }^{5}$ identificando los principios subyacentes al equilibrio de la estructura en cuestión. En la misma figura 4 (derecha), algunos adultos discuten el mismo problema durante una exposición científica, donde la comunicación pública de la ciencia era la tónica. Aunque en un primer momento la tarea puede parecer simplista, asemejándose al mero montaje de un rompecabezas, ella demanda importantes raciocinios que, en un momento dado de la historia desafió a importantes personajes de la historia, como Leonardo da Vinci (1452-1519), el primero en esbozar una teoría acerca de su funcionamiento, y Robert Hooke (1635-1703), quien formuló el problema en términos científicos más específicos.

En la figura 5 (izquierda), un grupo de alumnos, también de enseñanza primaria, dialogan (con la presencia de un mediador) en torno a un modelo dinámico del sistema solar (OCI) en una actividad escolar.

Los detalles del modelo se pueden ver en la figura 5 (centro). En la misma figura (derecha), varios adultos observan e indagan el mismo objeto durante una visita a una exposición científica interactiva dirigida al público general. Es importante observar que importantes cuestiones relacionadas con la teoría de la gravitación newtoniana fueron aquí objeto de reflexión y diálogo, movilizando la inteligibilidad y comprensión de esos diferentes públicos.

Destacamos, que la interacción con los diferentes materiales y la oportunidad de plantear cuestiones y discutir las hipótesis planteadas, proporcionan al público elementos esenciales de la educación científica.

\footnotetext{
${ }^{5}$ Se trata de una estructura (en forma de arco) formada por un semicírculo entero apoyado en dos extremos y cerrado por una sola pieza en forma de cuña. Este tipo de estructura comenzó a ser utilizada en la arquitectura Mesopotamia en el tercer milenio aC.
} 

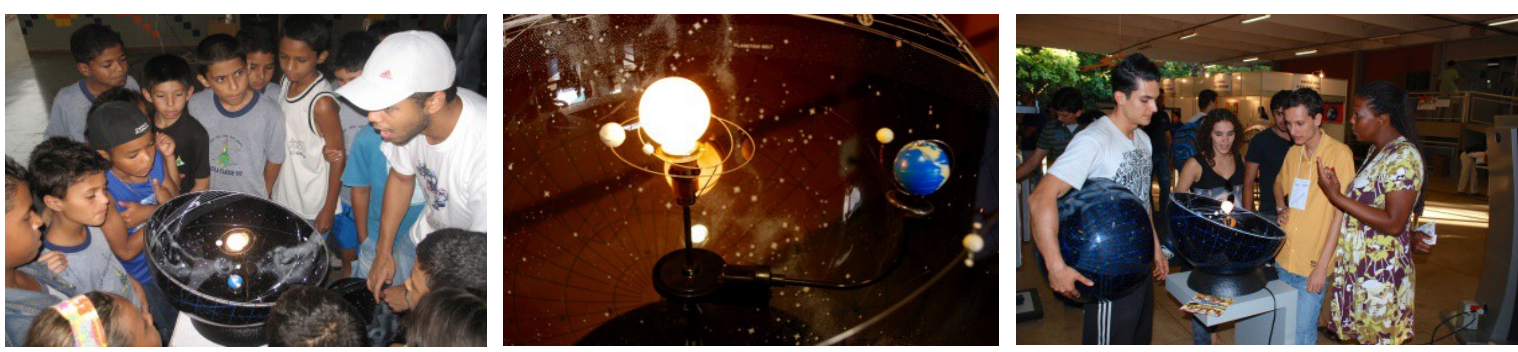

Figura 5. Alumnos de la educación básica (izquierda) y algunos adultos (derecha) en situaciones de aprendizaje investigativo.

Jerome Seymour Bruner (1915-2016), psicólogo estadounidense que destacó en el mundo de la educación por la defensa del aprendizaje como un proceso activo, fundado en bases cognitivas y eminentemente contextuales, desarrolló un conjunto de teorías sobre el aprendizaje, el lenguaje, el currículo, la pedagogía y la antropología que tuvieron una enorme influencia en los Estados Unidos y en Europa durante las décadas de 60, 70 y 80. Sus ideas inspiraron reformas curriculares que tuvieron gran impacto en el área de enseñanza de ciencias y matemáticas. Bruner enfatiza la necesidad de que los alumnos comprendan el propio proceso de descubrimiento científico, familiarizándose con las metodologías de las ciencias, asimilando sus principios y estructuras.

Bruner se mostró como un gran crítico de las llamadas metodologías expositivas y preconizó lo que posteriormente se conoció como «método del descubrimiento». Su defensa era que «al principio, el alumno debe poder resolver problemas, conjeturar, discutir de la misma manera que se hace en el campo científico de la disciplina» (Bruner 1965). En esta perspectiva, el alumno es colocado en situaciones de aprendizaje basándose en su propia experiencia y conocimiento anterior e interactuando con su ambiente, explorando y manipulando objetos, planteando preguntas, insertándose en controversias y/o realizando experimentos. Según Bruner, «la práctica de descubrir por si mismo enseña a adquirir información en un camino tal que la hace más fácilmente viable em la solución de problemas» (Bruner 1961).

El gran desafío aquí es movilizar a los estudiantes en su voluntad de descubrir y aprender. La figura 6 registra algunas situaciones cómo la mencionada anteriormente por Bruner.
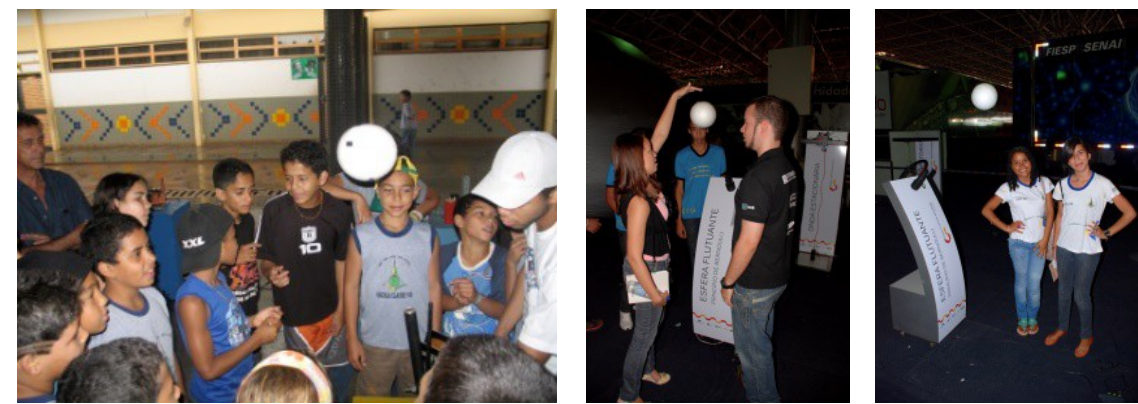

Figura 6. Esfera de porespán que flota, sostenida por la presión hidrodinámica.

En estas situaciones, estudiantes de enseñanza primaria y secundaria sugieren y discuten hipótesis para explicar cómo una esfera de porespán puede flotar sostenida por presión hidrodinámica producida por el flujo de aire en su superficie. El cuestionamiento, aliado a la observación y al trabajo investigativo y cooperativo, moviliza la comprensión de los alumnos acerca del fenómeno estudiado. Al protagonizar el proceso investigativo, se ven movilizados en su inteligibilidad y comprensión del mundo que los rodea. 

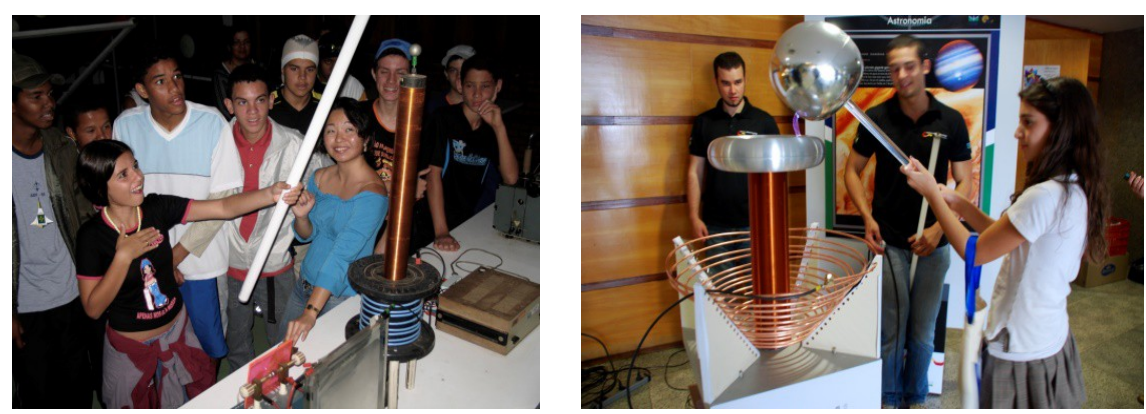

Figura 7. Alumnos de la escuela secundaria exploran los efectos de una bobina de Tesla en una exposición interactiva.

En la figura 7, los efectos de la acumulación de altas tensiones en una bobina de Tesla son explotados de forma lúdica e interactiva por alumnos de secundaria. Es importante enfatizar el hecho de que no se trata pura y simplemente de la realización de actividades prácticas de carácter demostrativo o ilustrativo, sino de un trabajo donde los estudiantes planifiquen su investigación, haciendo registros sistemáticos de sus observaciones, seleccionando variables relevantes en el proceso estudiado, controlando los valores de esas variables, operando equipos, etc.

Otro frente de acción es el proyecto «Planetario en la Escuela». Vemos en la figura 9 el planetario montado en patios internos de escuelas de la red de enseñanza.
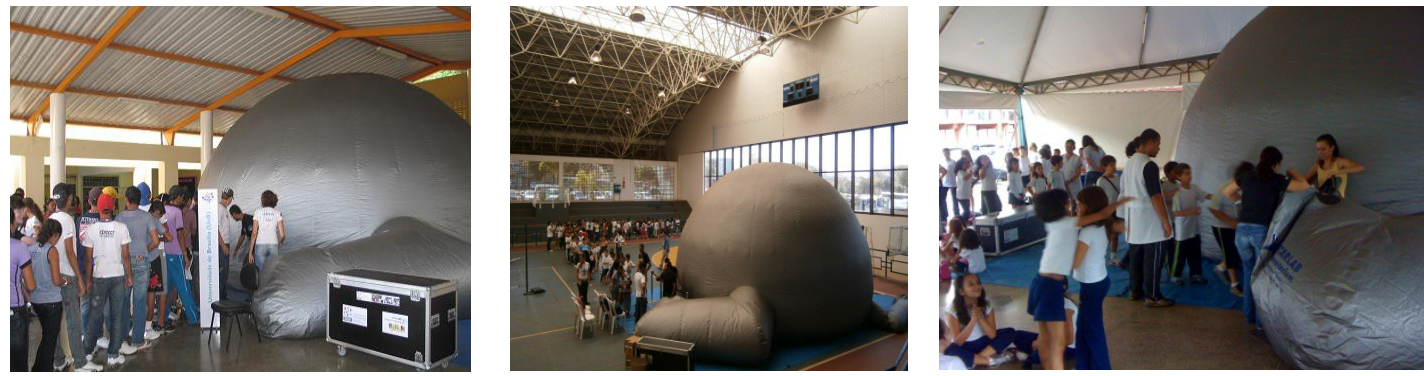

Figura 8. Planetario inflable en operación en la escuela.

Con un domo inflable (que admite entre 25-30 personas) y un proyector multimedia digital, asociado a un software específico, el cielo se proyecta en diferentes latitudes y husos horarios, permitiendo un diálogo rico, lúdico e interactivo con los visitantes sobre diferentes fenómenos astronómicos.

Nuestro trabajo se desarrolla con visitas sistemáticas a las escuelas de educación básica y comunidades de las diferentes regiones del Distrito Federal y entorno. Esto incluye, además de alumnos y profesores de enseñanza primaria y media, el público general. Son cientos de escuelas y miles de estudiantes de educación básica que ya han tenido acceso a las sesiones del planetario.

En la figura 9, se muestra el interior del domo inflable, con el equipo de mediadores (izquierda) ajustando el aparato, y un grupo de alumnos de enseñanza primaria de la red pública (derecha) demostrando su satisfacción y alegría en participar de la sesión.

La experiencia ha demostrado que, después de las sesiones en el planetario, los estudiantes demuestran mayor disposición y motivación para aprender astronomía (un contenido generalmente ausente del currículo de la educación básica) y, según testimonios de sus profesores, asumen un papel más activo en las clases de ciencias. Así, «Planetario en la Escuela» ha logrado diseminar conocimientos en el campo de la astronomía, promoviendo el espíritu investigativo de alumnos y profesores de la educación básica. 

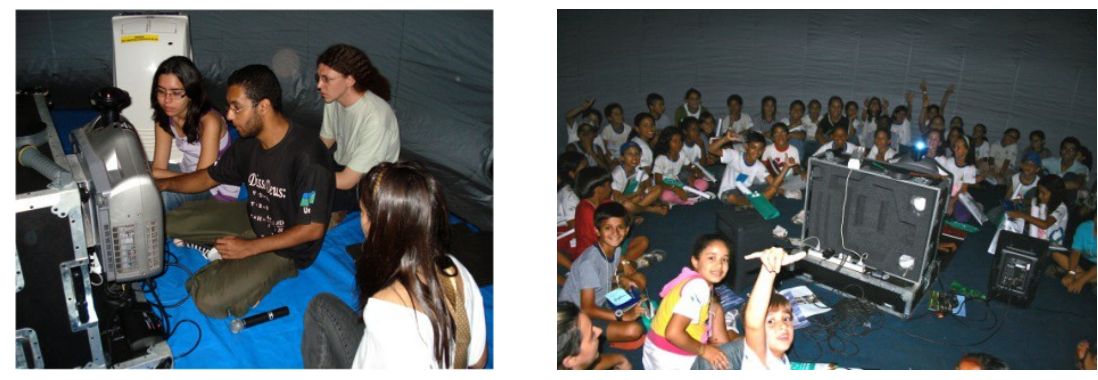

Figura 9. Interior del domo inflable del planetario.

De manera integrada y complementaria a las actividades del «Planetario en la Escuela», también se han utilizado exposiciones científicas itinerantes con gran éxito, como se muestra en las figuras 10 y 11.
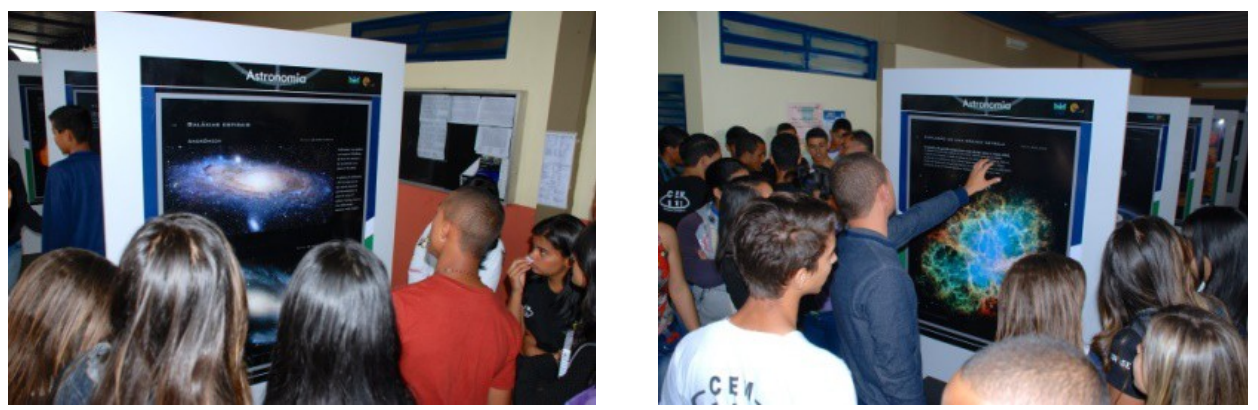

Figura 10. Centro de Enseñanza Media en Brasilia, recibe la exposición «Paisajes Cósmicos», con ocasión de su Encuentro de Arte, Ciencia y Cultura.

Nuestra asociación con las escuelas de educación básica, lejos de interferir en su rutina, acciones y actividades, busca siempre el establecimiento de una integración colaborativa en el campo pedagógico.

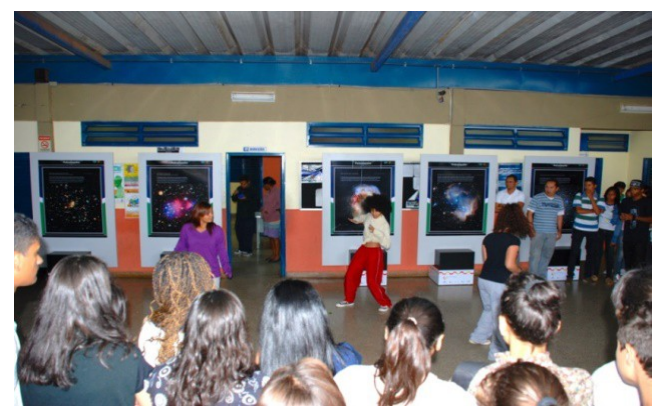

Figura 11. Presentación de un grupo de hip-hop durante un encuentro de arte, ciencia y cultura en un Centro de Enseñanza Media en Brasilia, DF, teniendo como escenario la exposición «Paisajes Cósmicos».

Nuestra experiencia ha demostrado, cada vez más, que el profesor de ciencias debe entender, como siendo su responsabilidad, la tarea de creación y coordinación de ambientes y situaciones de aprendizaje que potencien la interacción dialógica entre los alumnos en torno a objetos de conocimiento. En esta dirección, actividades basadas en situaciones que reivindican la exploración, la observación, la formulación y resolución de problemas, la previsión, el pensamiento crítico, la toma de decisiones y el diálogo deben formar parte del cotidiano de las clases de ciencias.

En este momento nuestra perspectiva es la de la continuidad del proyecto, buscando ampliarlo en el sentido de la capacitación de mediadores y profesores para el diseño de ambientes y 
situaciones de aprendizaje centradas en el estímulo a la curiosidad y al espíritu de investigación.

\section{Conclusiones}

A lo largo de este artículo, enfatizando la centralidad de la educación científica en la formación del ciudadano contemporáneo y defendiendo la necesidad de integración Universidad-Escuela en acciones de educación, divulgación y comunicación de la ciencia y de la tecnología, buscamos presentar brevemente lo que viene siendo desarrollado por el Instituto de Física de la Universidad de Brasilia, en asociación con algunas escuelas públicas de la capital federal y el Museo de la Ciencia y Tecnología de Brasilia. La idea-fuerza de las actividades aquí relatadas reside básicamente en el estímulo a la curiosidad y al espíritu de investigación de los participantes. Nuestra experiencia señala la necesidad de ampliación de las acciones de integración Universidad-Escuela, con el objetivo de potenciar la educación científica en la educación básica y la población en general.

\section{Agradecimientos}

Los autores agradecen al Ministerio de Ciencia, Tecnología e Innovación (MCTI) y al Consejo Nacional de Investigación ( $\mathrm{CNPq}$ ) el apoyo al desarrollo de este proyecto. Un agradecimiento especial se dirige a los estudiantes que actuaron como mediadores en estas acciones. A los alumnos y profesores de Educación Básica expresamos aquí también nuestro sincero agradecimiento por las alianzas y diálogos enriquecedores. Agradecemos también a los evaluadores, cuyas observaciones fueron centrales en la mejora de este artículo.

\section{Referencias}

Bachelard G. (1986) La formation de l'esprit scientifique, 13 ed. Paris: Libraire Philosophique J. Vrin.

Bruner J. (1961) The Act of Discovery. Harvard Education Review 30 (1), 21-327.

Bruner J. (1965) The Growth of Mind. American Psychologist 20, 1007-1017.

Freire P. (2016) Conscientização. São Paulo: Cortez Editora.

Freire P. (1973) ¿Extensión o Comunicación? Madrid: Siglo Veintiuno Editores

Massarani, L., Moreira I. C. (2002) Aspectos Históricos da Divulgação Científica no Brasil. pp. 43-64 en L. Massarani, I. C. Moreira, M. F. Brito (Orgs.), Ciência e Público: caminhos da divulgação científica no Brasil. 1ed. Rio de Janeiro: Casa da Ciência.

Massarani L., Moreira I. C. (2015).Science communication in Brazil: A historical review and considerations about the current situation. Anais da Academia Brasileira de Ciências 88, 1577-1595.

McConney A., Oliver M. C., Woods-McConney A., Schibeci R., Maor D. (2014) Inquiry, Engagement, and Literacy in Science: A Retrospective, Cross-National Analysis Using PISA 2006. Science Education 98 (6), 963-980.

OCDE (2016) PISA 2015 Assessment and Analytical Framework: Science, Reading, Mathematic and Financial Literacy. Paris: OECD Publishing.

Sagan C. (1997) The Demon-Haunted World. New York: Ballantine Books. (Edición en castellano (1997) El mundo y sus demonios. Barcelona: Ediciones B).

Wagensberg J. (2005) The "total" museum, a tool for social change. História, Ciências, Saúde Manguinhos 12 (Supplement), 309-321. 